\title{
Factores de riesgo para la preparación intestinal inadecuada en colonoscopia
}

\author{
Brenda A. de Anda-Martínez*, Martín Antonio-Manrique, Hugo J. Albores-Velázquez, \\ Sócrates Centellas-Hinojosa, J. Miguel Espinosa-González, M. Ángel Chávez-García y Jony Cerna-Cardona
}

Servicio de Endoscopia Gastrointestinal, Hospital Juárez de México, Ciudad de México, México

\begin{abstract}
Resumen
La preparación intestinal es un indicador de calidad de la colonoscopia. Una inadecuada preparación repercute en la tasa de detección de adenomas. Objetivo: Valorar los factores de riesgo asociados a una preparación intestinal inadecuada. Material y métodos: Se realizó un estudio prospectivo de enero a junio de 2018 en pacientes mayores de 18 años programados para colonoscopia en la Unidad de Endoscopia del Hospital Juárez de México. Se prepararon con polietilenglicol un día previo (4 litros) y se utilizó la escala de Boston de preparación intestinal. El análisis se realizó utilizando medias y desviación estándar (DE). Se realizó análisis de regresión con estadística no paramétrica para valorar correlación utilizando la rho de Spearman (r) ; se utilizó el software SPSS (versión 24). Resultados: Se realizaron 132 colonoscopias, se excluyeron 33. La edad promedio fue de 56.1 años (DE: \pm 15.29 ), $51.1 \%$ hombres vs. $48.9 \%$ mujeres. La principal indicación fue la hemorragia digestiva baja $(55 \%)$. La media de preparación intestinal fue de 7.35 (DE: \pm 0.96$)$. Los principales factores de riesgo con correlación significativa a mala preparación intestinal fueron: peso ( $r: 0.37 ; p=0.005)$, índice de masa corporal (IMC) ( $r: 0.46 ; p=0.005)$, índice cintura/cadera ( $r: 0.31 ; p=0.005)$, diabetes mellitus $(r: 0.41 ; p=0.05)$ y la ingesta alta de carnes rojas ( $r: 0.62 ; p=0.005)$. El resto de las variables medidas tiene pobre asociación con la adecuada preparación intestinal. Conclusión: Los factores de riesgo asociados a una preparación inadecuada de colon son IMC, índice cintural cadera, diabetes mellitus y la ingesta de carnes rojas.
\end{abstract}

Palabras clave: Preparación intestinal inadecuada. Escala de Boston. Factor de riesgo.

\section{Risk factors for inadequate bowel preparation on colonoscopy}

\begin{abstract}
Bowel preparation is an indicator of quality of colonoscopy. Inadequate preparation affects the detection rate of adenomas, prolong procedure times, and increased risk of complications. Objective: To assess the risk factors associated with inadequate bowel preparation. Material and methods: A prospective study was conducted from January to June 2018 in patients older than 18 years scheduled for colonoscopy in the Endoscopy Unit of the Hospital Juárez de México. They were prepared with polyethylene glycol the previous day (4 liters) and the Boston scale of intestinal preparation was used. The analysis was performed using means and standard deviation (SD). Regression analysis with non-parametric statistics was performed to assess correlation using Spearman's Rho (r), using the SPSS software (version 24). Results: 132 colonoscopies were
\end{abstract}

Correspondencia:

*Brenda Astrid de Anda-Martínez

E-mail: highhearted@ hotmail.com
Fecha de recepción: 27-05-2020

Fecha de aceptación: 29-05-2020 DOI: 10.24875/END.M20000177
Disponible en internet: 00-00-2000

Endoscopia. 2020;32(2):36-43 www.endoscopia-ameg.com 
performed, 33 were excluded. The average age was 56.1 years (SD: \pm 15.29 ), $51.1 \%$ men vs. $48.9 \%$ women. The main indication was lower gastrointestinal bleeding (55\%). The mean intestinal preparation was 7.35 (SD: \pm 0.96$)$. The main risk factors in which a significant correlation was observed with poor intestinal preparation were: weight ( $r: 0.37 ; p=0.005)$, body mass index (BMI) (r: 0.46; $p=0.005)$, waist/hip index ( $r: 0.31 ; p=0.005)$, diabetes mellitus ( $r: 0.41 ; p=0.05)$ and high intake of red meat $(r: 0.62 ; p=0.005)$. The rest of the measured variables have a poor association with adequate intestinal preparation. Conclusion: The risk factors associated with an inadequate preparation of the colon are BMI, waist/hip index, diabetes mellitus and the intake of red meat.

Key words: Inadequate bowel preparation. Boston scale. Risk factor.

\section{Antecedentes}

La colonoscopia en nuestro medio continúa siendo el estudio de elección para la valoración de la mucosa intestinal ${ }^{1}$. Una preparación intestinal inadecuada tiene, por tanto, un impacto negativo en su eficiencia ${ }^{2}$, disminuyendo la tasa de detección de adenoma y aumentando los costos, el retraso diagnóstico, los tiempos de procedimiento y el riesgo de complicaciones ${ }^{3}$.

La preparación ideal para la colonoscopia debe ser fiable, limpiar el colon de todo el material fecal de manera rápida, sin alteración macroscópica o histológica de la mucosa colónica ${ }^{4}$. La preparación intestinal ideal no debe causar incomodidad al paciente o cambios en líquidos o electrólitos y debe ser segura, conveniente, tolerable y económica ${ }^{5}$.

Los regímenes de preparación intestinal generalmente incorporan modificaciones en la dieta junto con catárticos orales. La mayoría comúnmente recomiendan dieta líquida clara el día previo a la colonoscopia ${ }^{6}$. Aunque los componentes individuales de las preparaciones intestinales varían ampliamente, la combinación de restricción dietética y los catárticos han demostrado ser seguros y efectivos para la limpieza colónica en colonoscopia?.

El uso de soluciones de polietilenglicol-electrólito (PEG-ELS) es uno de los métodos más comunes de limpieza del colon. Grandes volúmenes (4 litros) se han utilizado tradicionalmente para lograr un efecto catártico ${ }^{8}$. Aunque el esquema de 4 litros PEG-ELS no ha sido autorizado por la Food and Drug Administration (se aprueba la dosificación única), existe una amplia evidencia de que la más alta calidad en la preparación se logra usando PEG-ELS en dosis fraccionadas de 4 litros, y este se considera el criterio actual preparación estándar de colonoscopia ${ }^{9}$.

Se considera que la preparación es buena cuando permite explorar más del $90 \%$ de la mucosa o cuando permite la detección de pólipos de $5 \mathrm{~mm}$ de tamaño. Todo informe endoscópico debe reportar el grado de limpieza del colon ${ }^{10}$.
La escala de limpieza de colon de Boston (ELCB) subdivide el colon en tres segmentos: colon izquierdo, colon transverso y colon derecho. Cada segmento obtiene una puntuación de 0 a 3, dependiendo del grado de limpieza ${ }^{11}$. La puntación total se obtiene sumando la puntación de cada segmento. Esta escala permite estandarizar el grado de preparación intestinal. Entre sus ventajas se incluyen que es sencilla de utilizar, se encuentra validada y posee un alto grado de concordancia interobservador ${ }^{12}$.

Múltiples factores de riesgo han demostrado afectar de manera negativa la calidad de la preparación intestinal, entre ellos podemos encontrar: estreñimiento crónico, cirrosis hepática, cirugía abdominal o pélvica previa, polifarmacia, uso de opioides o antidepresivos tricíclicos, y factores relacionados con la inmovilización, como la edad, condición física, obesidad, enfermedad neurológica y hospitalización ${ }^{13,14}$.

Estudios recientes sugieren que los trastornos relacionados con el síndrome metabólico pueden dificultar la intubación cecal, teniendo como principales factores de estudio: diabetes mellitus (DM), hipertensión, dislipidemias, tejido adiposo visceral abundante, índice de masa corporal (IMC) alto e índice de circunferencia de la cintura aumentado ${ }^{15}$.

La investigación de factores predictivos en estudios bien diseñados es de vital importancia para identificar pacientes con alto riesgo de una limpieza intestinal inadecuada evitando endoscopias repetidas innecesarias y el riesgo de perder lesiones neoplásicas ${ }^{16,17}$.

\section{Objetivo}

Valorar los factores de riesgo asociados a una preparación intestinal inadecuada.

\section{Criterios de inclusión}

Se incluyeron todos los pacientes mayores de 18 años sometidos a una colonoscopia en la Unidad de 
Endoscopia del Hospital Juárez de México, entre enero y junio de 2018.

\section{Criterios de exclusión}

- Ingesta insuficiente de preparación intestinal (menos del $75 \%$ del volumen esperado).

- Hipertensión arterial no controlada (hipertensión arterial sistólica $[\mathrm{HAS}]>180 \mathrm{mmHg}$ y/o hipertensión arterial diastólica $>100 \mathrm{mmHg}$ ).

- Pacientes con antecedentes quirúrgicos que involucren colon/recto (colectomía, resección intestinal, derivación intestinal).

- Afecciones médicas graves como insuficiencia cardiaca, insuficiencia hepática aguda, enfermedad renal grave (tratamiento sustitutivo renal) o clasificación de la New York Heart Association III-IV.

- Embarazo o lactancia.

- Pacientes con dificultad para ingerir la preparación intestinal (demencia).

- Pacientes reprogramados después de una colonoscopia previa con preparación intestinal inadecuada.

- Negativa a firmar el formulario de consentimiento informado.

- Pacientes menores de edad (18 años).

\section{Pacientes y métodos}

El modelo fue construido con pacientes ambulatorios consecutivos que fueron programados prospectivamente para colonoscopia entre enero y junio de 2018. La limpieza se evaluó usando la ELCB.

La preparación intestinal estándar en nuestro centro se realiza mediante PEG 3350 administrado a partir de las 14:00 horas del día previo a la cita, con un volumen total de 4 litros, fraccionados en dosis de 250 mililitros. Todas las colonoscopias se realizan durante el turno matutino. Adicional a la preparación intestinal se indica dieta a base de líquidos claros dos días previos al estudio. La hoja de instrucciones de preparación intestinal incluye representaciones gráficas de cómo administrar la preparación y la dieta.

Los parámetros estudiados en relación con el grado de limpieza colónica (mediante la ELCB) fueron: edad, sexo, indicación, comorbilidades relacionadas (DM, HAS, hipotiroidismo, obesidad/sobrepeso, dislipidemia e insuficiencia hepática), ingesta de medicamentos, riesgo oncológico (historia personal y familiar de cáncer), tabaquismo, factores dietéticos, escala de Bristol, estreñimiento, cirugías previas (abdominales y pélvicas), nivel educativo, procedencia (ambulatorio/ hospitalizado) y tolerancia a la preparación intestinal. También se tomó en cuenta el índice cintura/cadera (ICC). Estas determinantes fueron valoradas por medio de un cuestionario que se aplicó a cada paciente previo al estudio (Fig. 1).

\section{Análisis estadístico}

Se realizó análisis descriptivo de las variables categóricas y de las variables numéricas para encontrar frecuencias empleando medias y desviación estándar. Se realizó análisis de regresión con estadística no paramétrica para valorar correlación utilizando la rho de Spearman. Para lograrlo se utilizó el software SPSS (versión 24).

\section{Resultados}

Se analizaron 132 colonoscopias, se excluyeron 33 casos: el $14.3 \%(n=19)$ con antecedentes quirúrgicos que involucraban colon/recto (colectomía, resección intestinal, derivación intestinal); el 3.7\% $(n=5)$ con ingesta insuficiente de la preparación intestinal $(<75 \%)$; el $3 \%(n=4)$ fueron diferidas por hipertensión mal controlada (HAS > $180 \mathrm{mmHg}$ y/o hipertensión arterial diastólica > $100 \mathrm{mmHg}$; el 2.2\% $(n=3)$ fueron pacientes con afecciones médicas graves, como insuficiencia cardiaca y enfermedad renal grave, y el $1.5 \%(n=2)$ eran pacientes menores de 18 años.

De las 99 colonoscopias incluidas, un $9 \%(n=9)$ fueron incompletas: seis por neoplasia estenosante $(66.6 \%)$, dos por falta de preparación (22\%) y una por causa técnica (11.1\%). En las colonoscopias incompletas por falta de preparación no se alcanzó el colon transverso en ningún caso.

En cuanto al nivel de la preparación intestinal (valorada mediante la puntuación total de la ELCB), presentó la siguiente distribución (puntuación total en la ELCB): de 0 a 3, 5 (5.5\%); de 4 a 5, 11 (12.2\%); de 6 a 7, 26 (28.8\%), y de 8 a 9, 48 (53.3\%). La media fue de 7,35 (desviación estándar [DE]: \pm 0.96 ) (Fig. 2).

La edad promedio de los pacientes estudiados fue de 56.1 años (DE: \pm 15.29 ). La distribución por sexo fue muy similar: $51.1 \%$ hombres vs. $48.9 \%$ mujeres. Más de la mitad de los pacientes tuvieron como indicación para realización de la colonoscopia hemorragia digestiva baja inactiva (50 pacientes, 55\%). El escrutinio de cáncer de colon $(25.5 \%)$ y la diarrea crónica $(18.8 \%)$ siguieron en orden de frecuencia. La principales comorbilidades que se observaron en la población estudiada 


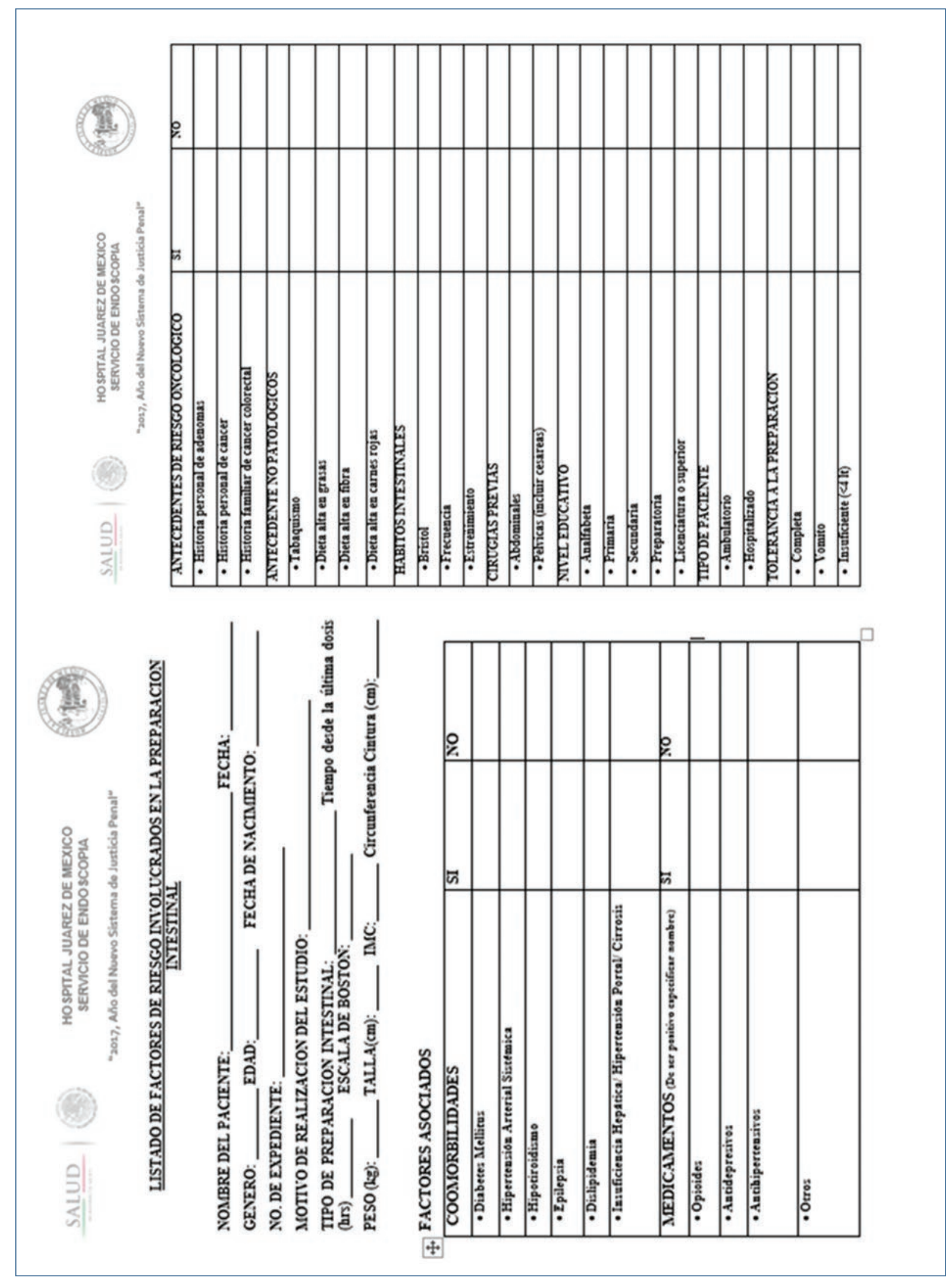

Figura 1. Hoja de recolección de datos. 


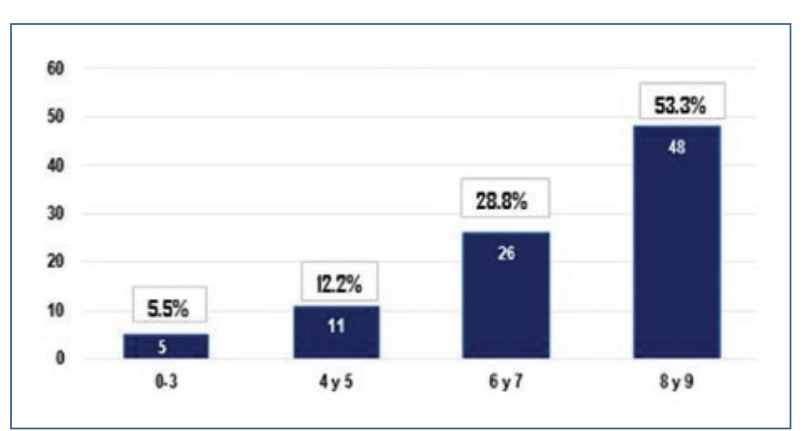

Figura 2. Nivel de preparación intestinal de acuerdo con la escala de Boston.

fueron DM (29 pacientes, 32.2\%) e hipertensión arterial (28 pacientes, 31.1\%). Los medicamentos más comúnmente observados fueron los antihipertensivos (31\%). Diecinueve pacientes contaban con historia personal de cáncer y 18 pacientes tenían algún familiar con cáncer colorrectal. En cuanto a los antecedentes personales no patológicos, una tercera parte de los pacientes (33\%) consumieron tabaco en algún momento de su vida, un $43.3 \%$ admitió una ingesta alta de grasas y un $41.1 \%$ de carnes rojas en su dieta. Cuarenta y siete pacientes tuvieron alguna cirugía abdominal previa (52.5\%), tanto convencional como laparoscópica. La mayor parte de la población contaban con nivel académico de primaria o secundaria. El $85 \%$ de las colonoscopias se realizaron a pacientes procedentes de su domicilio y solo un $5.5 \%$ a pacientes hospitalizados (Tabla 1).

El peso promedio de los pacientes incluidos fue de 71.8 kilogramos (DE: \pm 13.89 ) y la talla de 1.61 metros (DE: \pm 0.09 ). Llama la atención que el promedio del IMC en la población de estudio superó el límite normal (27.3, con DE: \pm 15.6$)$ y de la misma forma lo hizo el ICC, tanto para varones como para mujeres, lo cual se correlaciona con las estadísticas actuales para la población mexicana (Tabla 2).

Se observó una asociación entre el peso y la ELCB con una $r$ : $0.37(p=0.005)$, y el IMC con una $r$ : 46 $(p=0.005)$; el ICC también tiene relación con el grado de preparación, con una $r: 0.31(p=0.005)$, sin embargo en sus componentes la medición de la cadera tenía una correlación de $r$ : $0.39(p=0.005)$ y la medición de la cintura una $r$ : $0.46(p=0.005)$, teniendo una mejor correlación esta última.

El antecedente referido por los pacientes de consumo de dieta alta en carnes rojas presentó la correlación
Tabla 1. Análisis descriptivo de la población

\begin{tabular}{|c|c|}
\hline \multicolumn{2}{|l|}{ Análisis descriptivo de la población } \\
\hline $\begin{array}{l}\text { Número de pacientes } \\
\text { Edad } \\
\text { Sexo }\end{array}$ & $\begin{array}{c}n=90 \\
56(14 \pm 15.29) \\
n=46(51.1 \%) \text { varones }\end{array}$ \\
\hline $\begin{array}{l}\text { Indicación } \\
\text { Hemorragia digestiva } \\
\text { Escrutinio de cáncer de colon } \\
\text { Diarrea crónica }\end{array}$ & $\begin{array}{c}n=50(55 \%) \\
n=23(25.5 \%) \\
n=17(18.8 \%)\end{array}$ \\
\hline $\begin{array}{l}\text { Comorbilidades } \\
\text { Diabetes mellitus } \\
\text { Hipertensión arterial } \\
\text { Hipotiroidismo } \\
\text { Dislipidemia } \\
\text { Insuficiencia hepática }\end{array}$ & $\begin{array}{c}n=29(32.2 \%) \\
n=28(31.1 \%) \\
n=3(3.3 \%) \\
n=10(11.1 \%) \\
n=2(2.2 \%)\end{array}$ \\
\hline $\begin{array}{l}\text { Medicamentos } \\
\text { Antidepresivos } \\
\text { Antihipertensivos }\end{array}$ & $\begin{array}{c}\mathrm{n}=1(1.1 \%) \\
\mathrm{n}=28(31.1 \%)\end{array}$ \\
\hline $\begin{array}{l}\text { Riesgo oncológico } \\
\text { Historia personal de cáncer } \\
\text { Historia familiar de cáncer } \\
\text { colorrectal }\end{array}$ & $\begin{array}{c}\mathrm{n}=19(21.1 \%) \\
\mathrm{n}=18(20 \%)\end{array}$ \\
\hline $\begin{array}{l}\text { Antecedentes no patológicos } \\
\text { Tabaquismo } \\
\text { Dieta alta en grasas } \\
\text { Dieta alta en fibra } \\
\text { Dieta alta en carnes rojas }\end{array}$ & $\begin{array}{c}\mathrm{n}=30(33 \%) \\
\mathrm{n}=39(43.3 \%) \\
\mathrm{n}=22(24.4 \%) \\
\mathrm{n}=37(41.1 \%)\end{array}$ \\
\hline $\begin{array}{l}\text { Cirugía previas } \\
\text { Abdominales } \\
\text { Pélvicas }\end{array}$ & $\begin{array}{l}n=47(52.5 \%) \\
n=32(35.6 \%)\end{array}$ \\
\hline $\begin{array}{l}\text { Nivel educativo } \\
\text { Analfabeto } \\
\text { Primaria } \\
\text { Secundaria } \\
\text { Preparatoria } \\
\text { Licenciatura }\end{array}$ & $\begin{array}{c}n=10(11.1 \%) \\
n=29(32.2 \%) \\
n=29(32.2 \%) \\
n=16(17.8 \%) \\
n=4(4.4 \%)\end{array}$ \\
\hline $\begin{array}{l}\text { Tipo de estudio } \\
\text { Ambulatorio } \\
\text { Hospitalizado }\end{array}$ & $\begin{array}{c}n=95(94.4 \%) \\
n=5(6.6 \%)\end{array}$ \\
\hline
\end{tabular}

más fuerte entre todas las variables con una $\mathrm{r}$ : 0.62 $(p=0.005)$. Del resto de las variables, el antecedente de DM, con r: 0.41, estreñimiento con r: $0.34(p=0.005)$ y el antecedente de cirugía pélvica con una $r$ : 0.29 $(p=0.005)$.

En cuanto a la presencia de dolor al momento de la ingesta de la preparación, aunque se esperaría una mayor asociación con una mala preparación intestinal, tiene una correlación con una $r$ : 0.24 $(p=0.005)$.

El resto de las variables medidas, aunque presentaban alguna correlación, tienen una pobre asociación con la adecuada preparación intestinal (Tabla 3). 
Tabla 2. Variables antropométricos de la población de estudio

\begin{tabular}{|l|c|c|c|c|c|c|}
\hline \multicolumn{7}{|c|}{ Variables antropométricas de la población de estudio } \\
\hline & Peso & Talla & IMC & Cintura & Cadera & ICC \\
\hline Media & 71.8022 & 1.6162 & 27.3902 & 82.3667 & 88.5778 & 0.9280 \\
\hline Desviación Estándar & 13.89342 & 0.09934 & 4.25409 & 15.06499 & 12.62864 & 0.08530 \\
\hline
\end{tabular}

IMC: índice de masa corporal; ICC: índice cintura/cadera.

Tabla 3. Rho de Spearman

\begin{tabular}{|l|l|c|}
\hline Peso & $\begin{array}{l}\text { Coeficiente de correlación } \\
\text { Significación (bilateral) }\end{array}$ & 0.379 \\
\hline IMC & $\begin{array}{l}\text { Coeficiente de correlación } \\
\text { Significación (bilateral) }\end{array}$ & 0.463 \\
\hline Cintura & $\begin{array}{l}\text { Coeficiente de correlación } \\
\text { Significación (bilateral) }\end{array}$ & 0.565 \\
\hline Cadera & $\begin{array}{l}\text { Coeficiente de correlación } \\
\text { Significación (bilateral) }\end{array}$ & 0.392 \\
\hline ICC & $\begin{array}{l}\text { Coeficiente de correlación } \\
\text { Significación (bilateral) }\end{array}$ & 0.315 \\
\hline Diabetes mellitus & $\begin{array}{l}\text { Coeficiente de correlación } \\
\text { Significación (bilateral) }\end{array}$ & 0.002 \\
\hline Estreñimiento & Coeficiente de correlación & 0.416 \\
\hline Pélvicas & Significación (bilateral) & 0.345 \\
\hline & Coeficiente de correlación & 0.001 \\
\hline Dolor & Significación (bilateral) & 0.294 \\
\hline & Coeficiente de correlación & 0.005 \\
\hline
\end{tabular}

IMC: índice de masa corporal; ICC: índice cintura/cadera.

\section{Discusión}

El presente estudio nos permitió valorar el nivel de preparación intestinal observado en los pacientes sometidos a colonoscopia en nuestra unidad y los factores de riesgo asociados a la falla en la misma.

Un $83 \%$ de la población estudiada obtuvo 6 o más puntos en la ELCB, lo que hace aceptable la realización de colonoscopia. La preparación adecuada debe lograrse en al menos el $85 \%$, según la literatura, de todas las colonoscopias, ya que es un parámetro de calidad de esta ${ }^{18}$. Desafortunadamente existe una prevalencia del 20 al $25 \%$ de colonoscopias incompletas (sin canulación ileocecal), y la principal causa es una preparación intestinal inadecuada ${ }^{19}$. En nuestro estudio, al tener una baja prevalencia de mala preparación intestinal las variables estudiadas se presentan con poca frecuencia, por lo que el ampliar la población del estudio pudiera ayudar a mejorar las asociaciones entre estas y estudiar la probabilidad de falla.

En nuestro estudio los principales factores de riesgo en los cuales se observó una correlación significativa con la mala preparación intestinal fueron: IMC, la medición de la cintura, el ICC, la presencia de DM y la ingesta alta de carnes rojas. El resto de las variables, como cirugías pélvicas previas, estreñimiento y dolor abdominal tras de la ingesta de la preparación, si bien tienen una correlación, la asociación de estas con la mala preparación intestinal es débil, así como el resto de variables estudiadas, que se presentan en la tabla 1.

En cuanto a los efectos adversos secundarios a la preparación intestinal, solo el dolor abdominal tuvo una correlación con la falla en la preparación. La ingesta fue suficiente en la mayoría de los pacientes, por lo que el esquema de preparación utilizado en nuestro servicio mantiene buenos resultados y con un nivel de limpieza de colon aceptable.

En cuanto a las limitaciones del estudio podemos señalar que se tomó una muestra por conveniencia, ingresando a todos los pacientes que cumplieran con los criterios previamente establecidos, durante el periodo señalado. La recogida de datos se realizó mediante un cuestionario previo a la realización de la colonoscopia; si bien se diseñó con el objetivo de que fuera fácil en su llenado y comprensión, las variables de tipo nominal son subjetivas y se limitan a la percepción del paciente.

Aunque se han desarrollado múltiples formas de evaluar la calidad de la preparación intestinal, la ELCB es la más utilizada en nuestro medio, ya que tiene pocas variables que recordar y es fácilmente aplicable en la práctica clínica. Una puntuación global de al menos 6 puntos y una puntuación de al menos 2 por cada segmento colónico definen a la preparación colónica como adecuada. Consideramos la utilización de esta en nuestro servicio como una herramienta sumamente útil (Fig. 3). 


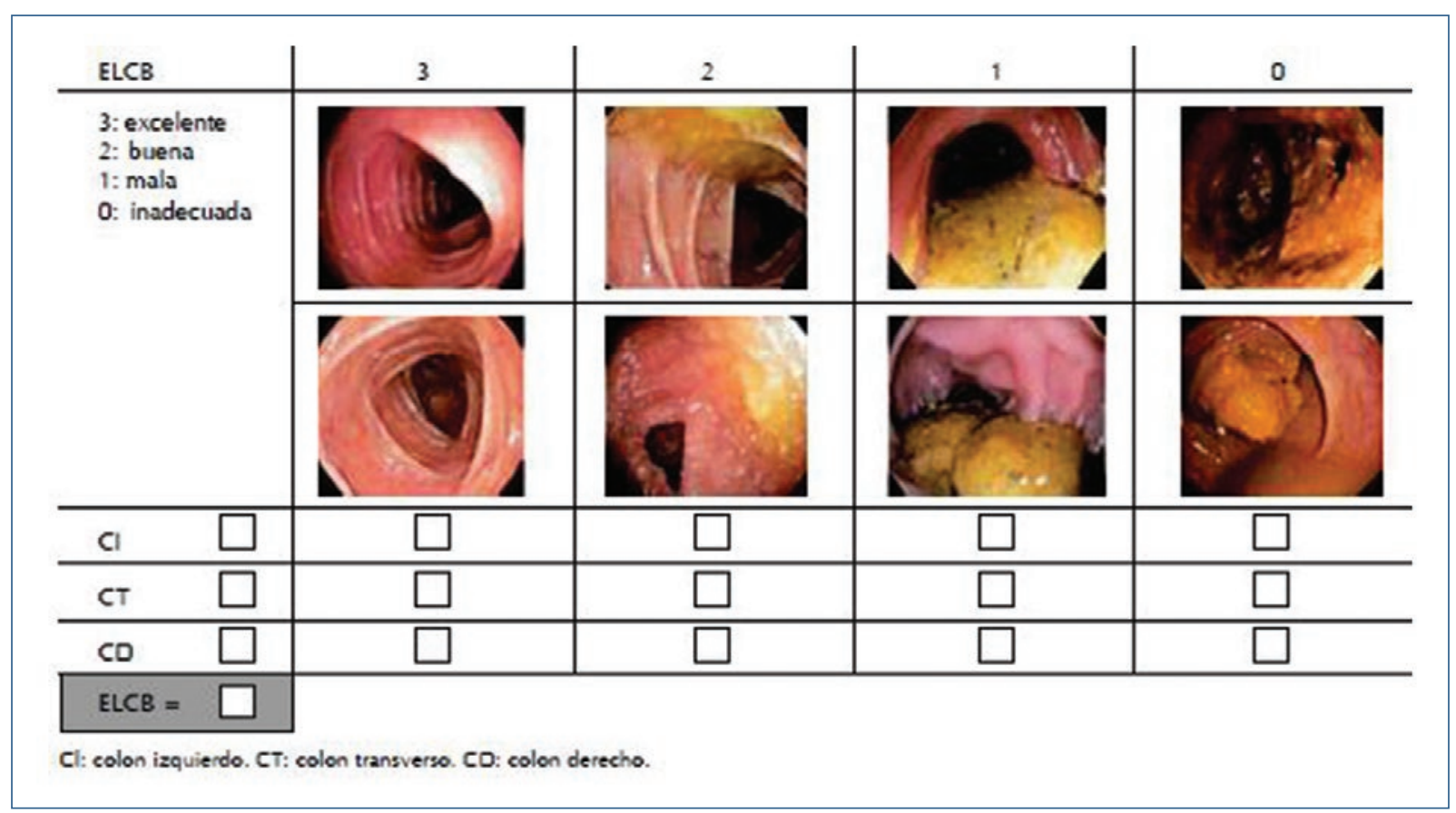

Figura 3. Escala de preparación de Boston.

ELCB: escala de limpieza de colon de Boston; CI: colon izquierdo; CT: colon transverso; CD: colon derecho.

\section{Conclusiones}

La falla en la preparación intestinal continúa siendo un reto para el endoscopista, ya que disminuye la calidad en la colonoscopia. Un adecuado abordaje del paciente previo al estudio, así como la elección de un adecuado esquema de preparación y la comprensión de este por parte del paciente son parámetros muy bien establecidos.

Las características de la población actualmente: mayor longevidad y por tanto un mayor riesgo de padecer comorbilidades, ingesta de medicamentos, sedentarismo, etc., nos obligan al estudio de nuevos factores de riesgo para falla en la preparación intestinal.

La DM, el sobrepeso/obesidad y los factores que conllevan un aumento en el riesgo cardiovascular (IMC e ICC altos) se presentan cada vez más en la población mexicana. En nuestro estudio tuvieron una correlación con preparación intestinal inadecuada, es necesario estudiar las causas subyacentes que expliquen dicho comportamiento.

Llama la atención la relación entre la falla en la preparación y el alto consumo de carnes rojas observada en nuestro estudio, posiblemente debido a que esta variable fue valorada de manera subjetiva; sin embargo, requiere un estudio más extenso que justifique los resultados.
Se sugiere llevar a cabo estudios donde se comparen diferentes poblaciones y sus factores de riesgo, para encontrar valores que pudieran tener una mayor correlación y poder integrar así una escala predictiva de falla a la preparación intestinal.

\section{Financiamiento}

Los autores no recibieron patrocinio para llevar a cabo este artículo.

\section{Conflicto de intereses}

Los autores declaran no tener ningún conflicto de intereses.

\section{Responsabilidades éticas}

Protección de personas y animales. Los autores declaran que los procedimientos seguidos se conformaron a las normas éticas del comité de experimentación humana responsable y de acuerdo con la Asociación Médica Mundial y la Declaración de Helsinki.

Confidencialidad de los datos. Los autores declaran que han seguido los protocolos de su centro de trabajo sobre la publicación de datos de pacientes. 
Derecho a la privacidad y consentimiento informado. Los autores han obtenido el consentimiento informado de los pacientes y/o sujetos referidos en el artículo. Este documento obra en poder del autor de correspondencia.

\section{Bibliografía}

1. Hassan C, Bretthauer M, Kaminski MF, Polkowski M, RembackenB, Saunders B, et al. Bowel preparation for colonoscopy: European Society of Gastrointestinal Endoscopy (ESGE) guideline. Endoscopy. 2013;45:142-50.

2. ASGE Standards of Practice Committee, Saltzman JR, Cash BD, Pasha SF, Early DS, Muthusamy VR, Khashab MA, et al. Bowel preparation before colonoscopy. Gastrointest Endosc. 2015;81:781-94.

3. Rex DK, Imperiale TF, Latinovich DR, Bratcher LL. Impact of bowel preparation on efficiency and cost of colonoscopy. Am J Gastroenterol. 2002;97:1696-700.

4. Johnson DA, Barkun AN, Cohen LB, Dominitz JA, Kaltenbach T, Martel $\mathrm{M}$, et al. Optimizing adequacy of bowel cleansing for colonoscopy: recommendations from the U.S. Multi-Society Task Force on Colorectal Cancer. Gastrointest Endosc. 2014;80:543-62.

5. Sweetser S, Baron TH. Optimizing bowel cleansing for colonoscopy. Mayo Clin Proc. 2015;90:520-6.

6. Nguyen DL, Jamal MM, Nguyen ET, Puli SR, Bechtold ML. Low-residue versus clear liquid diet before colonoscopy: a metaanalysis of randomized, controlled trials. Gastrointest Endosc. 2016;83:499-507.

7. Lorenzo-Zuniga V, Moreno-de-Vega V, Boix J. Preparation for colonoscopy: Types of scales and cleaning products. Rev Esp Enferm Dig. 2012;104:426-31.
8. Téllez-Ávila FI, Barahona-Garrido J, Osogobio-García S, López-Arce G, Camacho-Escobedo J, Saúl Á, et al. Quality of bowel preparation with 4-L PEG solution for colonoscopy in two Latin-American centers. Endoscopia. 2013;25:74-7.

9. Téllez-Ávila FI, Murcio-Pérez E, Saúl A, Herrera-Gómez S, Valdovinos-Andraca F, Acosta-Nava V, et al. Efficacy and tolerability of low-volume $(2 \mathrm{~L})$ versus single- $(4 \mathrm{~L})$ versus splitdose $(2 \mathrm{~L}+2 \mathrm{~L})$ polyethylene glycol bowel preparation for colonoscopy: randomized clinical trial. Dig Endosc. 2014;26:731-6.

10. Rex DK, Petrini JL, Baron TH, Chak A, Cohen J, Deal SE, et al. Quality indicators for colonoscopy. Am J Gastroenterol. 2006;101:873-85.

11. Lai EJ, Calderwood AH, Doros G, Fix OK, Jacobson BC. The Boston bowel preparation scale: A valid and reliable instrument for colonoscopy-oriented research. Gastrointest Endosc. 2009;69:620-5.

12. Calderwood AH, Jacobson BC. Comprehensive validation of the Boston bowel preparation scale. Gastrointest Endosc. 2010;72:686-92.

13. Lebwohl B, Wang TC, Neugut Al. Socioeconomic and other predictors of colonoscopy preparation quality. Dig Dis Sci. 2010;55:2014-20.

14. Antonio-Cisneros A, Antonio-Manrique M, Chávez-García MÁ, Hernández-Velázquez NN, Pérez-Valle E, Pérez-Corona T, et al. Factores pronósticos de preparación intestinal inadecuada en colonoscopia. Rev Hosp Jua Mex. 2016;83:75-9.

15. Cohen L. Advances in bowel preparation for colonoscopy. Gastrointest Endoscopy Clin N Am. 2015;25:183-97.

16. Rex DK. Bowel preparation for colonoscopy: Entering an era of increased expectations for efficacy. Clin Gastroenterol Hepatol. 2014;12:458-62.

17. Rostom A, Jolicoeur E. Validation of a new scale for the assessment of bowel preparation quality. Gastrointest Endosc. 2004;59:482-86.

18. Clark BT, Protiva P, Nagar A, Imaeda A, Ciarleglio MM, Deng Y, et al. Quantification of adequate bowel preparation for screening or surveillance colonoscopy in men. Gastroenterology. 2016;150:396-405.

19. Church J, Bast J, Elayi E, Xhaja X. Keeping the cecum clean: A randomized, prospective, placebo-controlled trial of loperamide as part of preparation for colonoscopy. Dis Colon Rectum. 2013;56:120-5. 